\title{
IMPLEMENTACIÓN DEL MODELO ESP (Inglés con Propósitos Específicos) EN LA UNIVERSIDAD AUTÓNOMA DE MANIZALES
}

\author{
Oskar Llano I. \\ Director Instituto de Idiomas \\ Carmenza Ríos $\boldsymbol{C}$. \\ Docente Instituto de Idiomas \\ Mónica Naranjo Ruiz \\ Docente Instituto de Idiomas
}

\section{RESUMEN}

La enseñanza del inglés en la UAM, se imparte con base en el modelo ESP; reconocido mundialmente como la enseñanza del Inglés con Propósitos Específicos. Este modelo se caracteriza porque el proceso enseñanza-aprendizaje del idioma se lleva a cabo con base en las necesidades específicas de los estudiantes; es decir, los contenidos se seleccionan de acuerdo con los programas que los estudiantes cursan; por lo tanto, los textos y materiales están relacionados con áreas específicas del conocimiento.

En la Universidad Autónoma de Manizales se ha implementado un modelo de enseñanza del inglés que integra los principios básicos del modelo ESP con elementos del Enfoque Comunicativo y de otros modelos, lo que ha dado como resultado una metodología ecléctica que integra todas las habilidades del lenguaje en una forma equitativa.

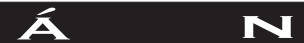

Universidad Autónoma de Manizales 
Los objetivos de este proyecto de investigación son: elaborar un marco teórico que sustente el modelo ESP utilizado para la enseñanza del inglés en la UAM, al mismo tiempo recopilar, sistematizar y analizar las experiencias obtenidas desde el inicio del trabajo con dicha metodología y, por último, reflexionar acerca de la experiencia enseñanza- aprendizaje con el propósito de determinar la forma más adecuada de retroalimentar el proceso. El trabajo se dividió en dos fases:

- Implementación del modelo ESP en la universidad Autónoma de Manizales

- Validación de la metodología ESP en la UAM

En este trabajo se presenta la primera fase, ya concluida, de la investigación. Para tal fin se realizaron las siguientes actividades:

- Revisión bibliográfica

- Descripción de la metodología utilizada para la enseñanza del inglés en la UAM y sus diferentes etapas, desde la creación del Departamento de Idiomas Extranjeros hasta hoy, convertido en Instituto.

- Recopilación de las memorias de los diferentes Talleres de Capacitación y de las actividades desarrolladas en cada uno de ellos.

- Análisis y sistematización de los Talleres recopilados.

- Análisis de resultados

Se determinó que la experiencia enseñanza-aprendizaje del inglés en la UAM cuenta con una base teórica sólida y con una retroalimentación constante del proceso a través de los Talleres de Capacitación docente. Estos últimos se clasificaron en cuatro categorías principales, que se encontraron directamente relacionadas con cuatro aspectos fundamentales del modelo ESP en la UAM: La Reflexión Metodológica, la Integración de las habilidades comunicativas, el Inglés con Propósitos Específicos (ESP) y la Evaluación. Por último se delimitó en el Instituto de Idiomas dos 
características relevantes: la formación en cuanto a la parte humana y la adquisición de los conocimientos profesionales.

\section{INTRODUCCIÓN.}

El modelo ESP (English for Specific Purposes), reconocido a nivel mundial como la Enseñanza del Inglés con Propósitos Específicos, se caracteriza porque en la clase de inglés se integran contenidos específicos relacionados con las áreas de conocimiento de los estudiantes; es decir, los contenidos se seleccionan teniendo en cuenta las necesidades académicas de los estudiantes, así por ejemplo, existe Inglés para Economía, Inglés para Ingeniería o Inglés para Odontología.

Tradicionalmente el ESP que se enseña en la mayoría de las universidades se enfoca únicamente hacia la compresión de lectura o simplemente hacia la traducción de textos para lo que, además, no se le proporciona al estudiante la formación adecuada, y se excluyen totalmente las demás habilidades comunicativas del idioma (Escucha, Habla, Escritura). A diferencia, en la universidad Autónoma de Manizales se ha implementado un modelo de enseñanza-aprendizaje del inglés que integra los principios básicos del modelo ESP con elementos del enfoque comunicativo y de otros modelos, lo que ha dado como resultado una metodología ecléctica que integra todas las habilidades del lenguaje en una forma equitativa y que además considera la "Traducción" como una quinta habilidad comunicativa, ya que ésta no sólo tiene gran relevancia como instrumento transmisor de cultura y conocimiento sino que es parte inherente de la comunicación en cualquier idioma.

Tanto la integración de elementos del enfoque comunicativo, como la consideración de la traducción como una habilidad comunicativa han posesionado nuestra metodología como un modelo exclusivo para la enseñanza del inglés en esta universidad, el cual a través de diez años, ha logrado que los estudiantes se concienticen de la importancia que tiene el estudio del inglés, no sólo a nivel general 
sino a nivel profesional, pues el estudio del idioma tiene como contexto materiales que son interesantes y relevantes para su formación profesional, lo que constituye un elemento motivante.

A pesar de que tanto los aspectos mencionados previamente como la capacitación-docente han sido elementos constantes y muy característicos del Departamento de Idiomas Extranjeros (hoy Instituto de Idiomas), no existía hasta el momento un documento que recopilara la experiencia, lo cual dio paso al surgimiento de este proyecto; por otro lado, también se hace necesario proporcionar un soporte teórico y validar el trabajo con dicho modelo.

Esta primera fase constituye la recopilación de la información concerniente a la enseñanza del inglés en la UAM desde su inicio hasta hoy, así como una descripción de la metodología con la cual se enseña el ESP en esta universidad, también se incluyen los aspectos más relevantes de la misma, el trabajo realizado con las cinco habilidades comunicativas, la recopilación y sistematización de los temas tratados en los talleres de capacitación y su pertinencia frente al proceso de enseñanza aprendizaje del inglés en la UAM.

Durante esta fase preliminar se pudo determinar que el modelo ESP en la UAM consta de una base teórica sólida y de una retroalimentación constante del proceso que se lleva a cabo. Por otro lado fue posible clasificar los temas tratados en los talleres de capacitación dentro de cuatro categorías, las cuales a su vez apuntan hacia cuatro aspectos característicos de la metodología empleada para llevar a cabo el proceso de enseñanza-aprendizaje del inglés en la UAM.

\section{DISEÑO Y METODOLOGÍA DE LA INVESTIGACIÓN}

Tipo de estudio: Sistematización de Experiencia

Como se mencionó antes, la capacitación ha sido un elemento constante en el trabajo realizado por el Departamento de Idiomas 
Extranjeros, pero sólo a partir de 1999 se empezó a realizar la recopilación escrita sobre dicha capacitación. Fue así como en el año 1999, se inició la elaboración de una especie de memoria para cada taller, en la cual se registran los aspectos acerca de los cuales los docentes discuten y reflexionan durante cada encuentro. Estas memorias junto con los materiales que se habían utilizado en cada taller fueron la base para la sistematización de la experiencia. Es valioso resaltar que también fue necesario rescatar los materiales y documentos utilizados en los talleres de los años anteriores y se elaboró con la ayuda de fuentes personales directas (docentes antiguos) las memorias para cada uno de ellos (o al menos un resumen pequeño acerca del tema tratado).

Este es un proyecto de investigación que combina además el elemento cualitativo ya que se desarrolla fundamentalmente con base en el análisis de los datos obtenidos mediante las memorias de los talleres de capacitación, análisis que además dio como resultado la determinación de ciertas variables dentro de dichos talleres.

Para el desarrollo de la investigación se llevó a cabo la siguiente línea de trabajo:

\section{Reflexión inicial}

Durante esta etapa se determinó la necesidad de plasmar en forma escrita la experiencia metodológica del Departamento de Idiomas Extranjeros, desde su creación en 1992.

\section{Planeación}

El siguiente paso fue determinar los objetivos de la investigación y la metodología para alcanzarlos.

\section{Acción}

- Revisión bibliográfica acerca de las metodologías utilizadas para la enseñanza del inglés desde el inicio de su expansión a través de diferentes países hasta llegar al modelo ESP.

- Descripción de la metodología utilizada para la enseñanza del

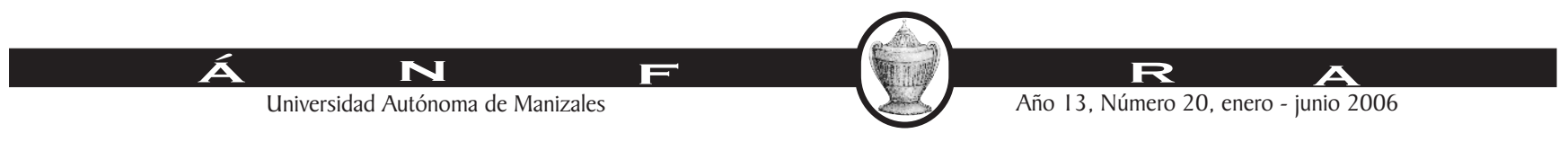


inglés en la UAM y sus diferentes etapas, desde la creación del Departamento de Idiomas Extranjeros hasta hoy.

- Recopilación de las memorias de los diferentes talleres de capacitación y de las actividades desarrolladas en cada uno de ellos.

- Análisis y sistematización de los talleres recopilados.

- Análisis de resultados

Para la realización de estas actividades se conformaron dos grupos de trabajo, el primero de ellos se encargó de la revisión bibliográfica del tema, para lo cual se realizó una búsqueda exhaustiva a través de diferentes bibliotecas y de la red (Internet), acerca del origen de la enseñanza del Inglés Como Lengua Extranjera (EFL) hasta llegar al modelo ESP. Esta búsqueda se efectuó con el fin de elaborar la fundamentación teórica del modelo ESP utilizado para la enseñanza del inglés en esta universidad.

El segundo grupo inició la recopilación de la experiencia del Departamento desde su creación, para esto se llevó a cabo la recopilación de las memorias de los diferentes talleres de capacitación, así como la lectura de diferentes documentos acerca del modelo ESP en la UAM. Cada grupo contó con una coordinación y con el director del Departamento como asesor.

Posteriormente se efectuó la fusión de los dos trabajos, para lo cual se nombró una sola coordinadora quien se encargó de organizar toda la información obtenida hasta ese momento, conformar el cuerpo del trabajo y continuar con la recopilación, análisis y sistematización de los talleres de capacitación.

Por último se llevó a cabo la sistematización de los talleres de capacitación a través de la correlación de los mismos con la metodología del Departamento. 


\section{Reflexión final}

Esta última etapa se desarrolló con base en la sistematización de los talleres de capacitación, durante ésta se determinaron las fortalezas y falencias de la capacitación docente en el Departamento de Idiomas Extranjeros de la UAM, lo que a su vez permitió la elaboración de las conclusiones y de algunas recomendaciones.

La recolección de la información se realizó básicamente a través de la consulta bibliográfica tanto en bibliotecas como en Internet y se contó con el valioso apoyo de los docentes que han laborado durante largo tiempo en el Departamento de Idiomas como fuentes personales directas de información.

\section{EL MODELO ESP EN LA UAM}

Durante la elaboración del marco teórico y conceptual del proyecto de investigación se determinó en primer lugar, la existencia de cuatro aspectos que caracterizan la metodología ESP en la UAM:

\section{LA METODOLOGÍA ECLÉCTICA:}

El modelo ESP en la cual se basa el proceso de enseñanza aprendizaje de la UAM, toma específicamente elementos de los siguientes modelos y enfoques: enfoque comunicativo, ContentBased Teaching (Enseñanza Basada en Contenido), Experiential Learning (Aprendizaje Experiencial), TaskBased Teaching (Enseñanza Basada En Tareas), Método AudioLingual (MAL), Método AudioVisual, Enfoque Cognitivo, WholeLanguage (Lenguaje Total).

\section{LA INTEGRACIÓN DE LAS HABILIDADES COMUNICATIVAS:}

El objetivo primordial del Departamento de Idiomas de la UAM es desarrollar la competencia comunicativa en lengua extranjera en los estudiantes y para este fin, se les proporcionan clases donde están constantemente expuestos al idioma objetivo; es decir las clases se

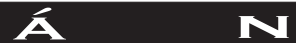

Universidad Autónoma de Manizales 
desarrollan $100 \%$ en inglés lo que además permite el desarrollo constante de la escucha y el habla.

Para el desarrollo de la escucha se busca, igual como sucede en el proceso natural de aprendizaje de la primera lengua, que los estudiantes estén permanentemente expuestos a la lengua hablada y paulatinamente desarrollen el habla. Para tal fin, las clases de inglés no sólo se desarrollan en inglés sino que también se realizan actividades de escucha ya sea a través de videos, audio-casettes o CDs. Como un factor relevante se tiene siempre en cuenta, que el material de escucha utilizado contenga un lenguaje natural y en lo posible sea auténtico; es decir que no haya sido diseñado con fines pedagógicos, por ejemplo: comerciales, fragmentos de películas o programas de radio o televisión. También hacen parte del material utilizado, los cassettes complementarios del texto de estudio utilizado para la enseñanza del inglés en la UAM. Se trata siempre de promover la producción del estudiante y se excluyen totalmente los ejercicios de repetición o de simple memorización.

Ya que el habla está directamente ligada a la escucha a medida que el estudiante está expuesto constantemente al inglés en forma oral, éste va desarrollando bases y estableciendo modelos a seguir para lograr su producción oral en inglés. Por esto a través del desarrollo de esta habilidad no sólo se expone al estudiante al idioma sino que se desarrollan actividades que exijen un desempeño oral, las cuales pueden ir desde simple respuestas a preguntas hechas por el docente, hasta debates, foros o presentaciones orales, dependiendo del nivel que estén cursando los estudiantes. Es importante destacar que en todas estas actividades siempre se trata de integrar los contenidos relacionados con el área de conocimiento de los estudiantes.

No podemos dejar de lado el desarrollo de la lectura y de la escritura. En el caso de la primera, el Departamento de Idiomas busca el desarrollo de la lectura comprensiva lo que se lleva a cabo tanto a través del material diseñado por los docentes, como de las lecturas y

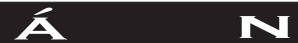

Universidad Autónoma de Manizales 
ejercicios de comprensión contenidos en el texto guía. A la hora de diseñar el material para la comprensión de los textos de lectura, se busca siempre que el material esté contextualizado con el área de los estudiantes, que el nivel y el vocabulario sean adecuados para su nivel y que los temas además de ser interesantes y relevantes para ellos, también sean previamente conocidos en su primera lengua o al menos posean un conocimiento general sobre ellos.

En cuanto a la escritura, habilidad que al igual que las demás es desarrollada desde primer nivel, se busca guiar a los estudiantes en un proceso secuencial que se inicia con la construcción de oraciones hasta llegar a la escritura de párrafos. El propósito primordial es desarrollar la producción de los estudiantes y para ello se les dan modelos a seguir y se excluyen los ejercicios de tipo mecánico. También se fomenta la autocorrección aunque siempre se cuenta con la asesoría del docente.

Un aspecto muy relevante para la enseñanza del ESP en la UAM y en particular para el desarrollo de las diferentes habilidades comunicativas es la motivación. Los docentes son conscientes que es necesario proveer estímulos para que el estudiante se interese por el aprendizaje del idioma, lo que se logra paso a paso, con el simple hecho de interesarlo por cada actividad realizada en la clase. Es por ello que para el desarrollo de cualquiera de las habilidades mencionadas anteriormente, siempre se tienen en cuenta tres fases fundamentales: PRE, DURANTE y POST.

La PRE-fase comprende las actividades realizadas previamente a la actividad central, ya sea escucha, habla, etc. Durante esta fase se pone al estudiante en contacto con el tema a tratar, se averigua su conocimiento previo del tema y en general se proveen las herramientas necesarias para el desarrollo y entendimiento de la siguiente fase.

La siguiente fase se denomina DURANTE; ésta consiste en el desarrollo de la actividad directamente relacionada con la habilidad

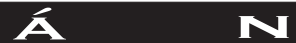

Universidad Autónoma de Manizales 
que es objetivo central.

Finalmente vienen las actividades posteriores a la actividad central, durante las cuales el estudiante realiza ejercicios de producción, reflexión o análisis acerca del tema tratado en las fases anteriores. En esta última fase, es donde el estudiante se concientiza de la aplicabilidad y la importancia del tema y el docente a su vez, tiene la oportunidad de evaluar el grado de asimilación del tema por parte de sus estudiantes.

Finalmente tenemos la Traducción que como se dijo en la primera parte de este documento, es inherente a las demás habilidades comunicativas. Así la traducción es parte fundamental para el desarrollo de las cuatro habilidades comunicativas tradicionales, y en el caso de la UAM, se pretende lograr que los estudiantes hagan uso de la traducción intralingual; es decir que desarrollen la capacidad de codificar y decodificar mensajes en inglés. No se trata de que los estudiantes hagan traducciones de una lengua a otra sino de que empleen la misma lengua extranjera para entender y aprehender todo la información que reciben en esa misma lengua. Por esto siempre se hace uso de recursos tales como la mímica, la ejemplificación o de diferentes medios audio-visuales para explicar cualquier tipo de vocabulario o idea que sea desconocida para el estudiante.

El desarrollo de las diferentes habilidades comunicativas se da en forma secuencial partiendo de la escucha hasta llegar a la escritura. Así se le brinda la oportunidad a los estudiantes de sumergirse en el idioma durante las clases, a través de diversas actividades que buscan desarrollar todas las habilidades lingüísticas mientras el estudiante refuerza temas concernientes a su área de conocimiento. Así las clases modelan lo que sucede en la vida real y los estudiantes tienen la oportunidad de desempeñarse a nivel receptivo; mientras escuchan y leen, y a nivel productivo; cuando hablan y escriben.

Ninguna habilidad del lenguaje debe ser excluida o aislada de las

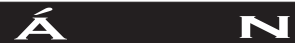

Universidad Autónoma de Manizales 
demás ya que es el conjunto de todas lo que nos permiten la interacción: recibir y enviar mensajes y es ése el objeto de la comunicación, además, una habilidad refuerza otra; así hablamos porque recibimos modelos cuando escuchamos y escribimos porque podemos leer.

En general con esta integración se busca esencialmente la funcionalidad del lenguaje, en donde los contenidos y las formas lingüísticas interactuan para dar como resultado la comunicación.

\section{LA ENSEÑANZA COOPERATIVA:}

La enseñanza del inglés en la UAM está caracterizada no sólo por su metodología ecléctica o por la integración de las habilidades comunicativas, sino por la uniformidad y organización entre los docentes. Así, a pesar de diversidad de programas académicos y por lo tanto de perfiles en los estudiantes de los diferentes programas, los docentes del Departamento de Idiomas realizan un trabajo cooperativo; de equipo, para lo cual se realizan reuniones semanales por niveles, durante las cuales se planean las actividades y se diseñan los materiales con los cuales se va a trabajar. Por supuesto, cada docente diseña de acuerdo con su área pero se busca que a la hora de diseñar y escoger los temas relacionados con la lengua exista una uniformidad metodológica. Así por ejemplo, todos los estudiantes de nivel I en todas las carreras están expuestos a las mismas actividades y a materiales con estrategias similares, aunque cada grupo tiene diferentes contextos de aprendizaje, en este caso sus campos de estudio.

Este trabajo por equipos también permite el establecimiento y perduración de una metodología uniforme que evita traumatismos cuando un estudiante pasa de un nivel a otro; todos los docentes buscan los mismos objetivos: desarrollar habilidades comunicativas en el estudiante y proporcionarle conocimientos relacionados con su área de estudio.

LA ENSEÑANZA REFLEXIVA:

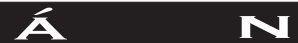

Universidad Autónoma de Manizales 
La capacitación de docentes tiene que ser indiscutiblemente el eje de cualquier programa educativo, ya que existe muy poca probabilidad de éxito sino se cuenta con docentes preparados para satisfacer las necesidades especificas de los estudiantes. Es por eso que desde la creación del Departamento de Idiomas, la dirección del Departamento ha buscado siempre la capacitación de su personal docente así como la optimización de los resultados obtenidos. Por esto semanalmente se realizan los "talleres de capacitación", durante los cuales los docentes no sólo se capacitan sobre diversos temas relacionados con la docencia, sino que comparten experiencias y reflexionan sobre el que hacer diario en el aula de clase. Así, la finalidad tanto de los talleres como de las reuniones por nivel es la optimización de los procesos docentes mediante la elaboración y análisis grupal de diversas actividades pedagógicas, tales como la preparación de clases, el acto magistral (la clase) y la evaluación de actividades.

En conclusión la reflexión es un elemento constante en el proceso enseñanza aprendizaje del inglés y un elemento clave a la hora de ofrecer calidad a nuestros estudiantes.

Además de los cuatro aspectos mencionados antes y que son tal vez los más relevantes de la metodología ESP en la UAM, el contenido enseñado en las clases de inglés también es fundamental para la funcionalidad y efectividad de la enseñanza del idioma y es lo que hace que el método utilizado conserve siempre su esencia como ESP. Para ello, los contenidos integrados en la clase de inglés son seleccionados cuidadosamente y se tienen en cuenta factores tan importantes como el conocimiento previo que los estudiantes poseen en su lengua materna acerca del tema; para ello se tienen en cuenta los planes de estudio de las diferentes áreas que el estudiante cursa o ha cursado. Así mismo se busca siempre que los contenidos no sólo sean conocidos por los estudiantes sino que sean interesantes y relevantes para ellos.

La metodología ESP utilizada en la UAM presenta entonces ventajas diversas para lograr un aprendizaje efectivo por parte de los estudiantes, pues éstos no sólo aprenden el idioma en una forma comunicativa y

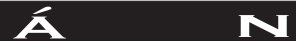

Universidad Autónoma de Manizales

\section{R}

Año I3, Número 20, enero - junio 2006 
natural, sino que aprenden sobre temas de su interés; lo que además proporciona un contexto para el aprendizaje de la lengua, así el contenido ayuda al aprendizaje y práctica de la lengua y la lengua ayuda a entender el contenido.

Con base en las reflexiones hechas por los docentes del Departamento también se ha concluido que:

- El trabajo colaborativo entre docentes y la integración de las habilidades lingüísticas son aspectos claves para el éxito del proceso enseñanzaaprendizaje.

- Las clases deben caracterizarse por la variedad de actividades y recursos audiovisuales empleados.

- Debe existir relación entre los contenidos enseñados y las funciones lingüísticas estudiadas en la clase de inglés.

- Es necesario realizar trabajo individual y grupal.

- El aprendizaje de la lengua - objetivo debe hacerse en esa misma lengua.

La evaluación es también parte fundamental en el proceso enseñanzaaprendizaje del inglés en la UAM, y esa es la razón por la que para evaluar a un estudiante se tienen en cuenta su nivel de aprehensión y manejo de las nociones y funciones lingüísticas tratadas en la clase, su desempeño en cada una de las habilidades lingüísticas y su asimilación de los contenido relacionados con su campo de conocimiento, tratados en clase. Se busca primordialmente que el estudiante logre comunicarse efectivamente en la lengua objetivo.

Así, la evaluación en las clases de inglés es el resultado de la combinación de las observaciones y juicios que el docente hace respecto al desempeño de sus estudiantes mientras interactúan entre ellos o con el docente (en inglés) y del desempeño de los estudiantes en otras actividades y tareas relacionadas con la lectura y la escritura.

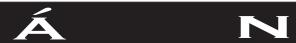

Universidad Autónoma de Manizales

\section{$\mathbf{R}$}

Año I3, Número 20, enero - junio 2006 


\section{RESULTADOS}

Durante la elaboración de este trabajo fue posible observar que la experiencia enseñanza- aprendizaje del inglés en la UAM cuenta con una base teórica sólida y con una retroalimentación constante del proceso que se lleva a cabo, elementos que han enriquecido el modelo ESP utilizado en la UAM, y que a su vez han impedido la fosilización de los conocimientos de los docentes y del modelo como tal.

La retroalimentación ha permitido además la adaptación permanente del modelo según las necesidades de los estudiantes y del medio, esto se refleja claramente a través de los cambios descritos, respecto a las diferentes fases por las que ha pasado el modelo ESP en la UAM.

De otro lado también fue posible determinar cuatro aspectos fundamentales del modelo ESP en la UAM, los cuales además fueron relacionados con los talleres de capacitación docente lo que arrojó cuatro categorías : la Reflexión Metodológica, el Desarrollo de las Habilidades Comunicativas, el Inglés con Propósitos Específicos (ESP) y la Evaluación en el aula de clase.

Pero más que las cuatro categorías obtenidas a través de la sistematización de los talleres de capacitación, se observó que en el trabajo realizado por el Departamento de Idiomas priman dos aspectos que son fundamentales para el proceso de enseñanza - aprendizaje de los estudiantes de hoy, sin importar su área de estudio: La formación en cuanto a la parte humana y la adquisición de los conocimientos profesionales. Esto se refleja claramente en los talleres ubicados en la categoría 1 (Reflexión Metodológica) los cuales están directamente relacionados con estos dos aspectos.

Los talleres incluidos en esta categoría fueron dirigidos a la sensibilización del docente, no sólo para lograr una mejor interrelación con los estudiantes sino para lograr despertar en estos últimos toda esa parte humana que muchas veces se deja de lado porque prima la necesidad de adquirir los conocimientos necesarios para aprobar un curso. Es importante aclarar en este punto que aunque estos talleres estuvieron directamente relacionados 
con el aspecto de la sensibilización, en general durante estos encuentros el saber entender al estudiante como el ser humano que es, fue un elemento constante e implícito.

Las tres categorías restantes (Habilidades Comunicativas, ESP, Evaluación) apuntan más hacia el fomento del conocimiento profesional de los docentes, aunque como se dijo antes, la reflexión y por lo tanto la parte de sensibilización son una constante. Dentro de estas categorías se observó que la capacitación docente ha estado más dirigida hacia la reflexión metodológica que hacia las demás áreas, lo cual constituye una debilidad, ya que tanto el trabajo con el ESP como el desarrollo e integración de las habilidades comunicativas son pilares del modelo ESP en esta universidad, es por ello que estos aspectos deben estudiarse con más profundidad.

Por último la Evaluación es otro aspecto en el que es necesario enfatizar más, ya que ésta constituye un punto importante de apoyo para el desarrollo y la retroalimentación constante del proceso de enseñanza aprendizaje que se lleva a cabo.

\section{CONCLUSIONES}

- La capacitación docente se ha dirigido más hacia la reflexión metodológica que hacia los demás aspectos .

- Los talleres de capacitación docente realmente apuntan hacia los objetivos del Departamento de Idiomas Extranjeros y hacia la metodología utilizada para la enseñanza del inglés en la UAM. Por esto fue posible clasificarlos de acuerdo con aspectos característicos de esta metodología.

- La capacitación docente ha sido una constante en el Departamento de Idiomas Extranjeros de la UAM.

- El modelo ESP empleado para la enseñanza-aprendizaje del inglés en la UAM tiene una base teórica sólida.

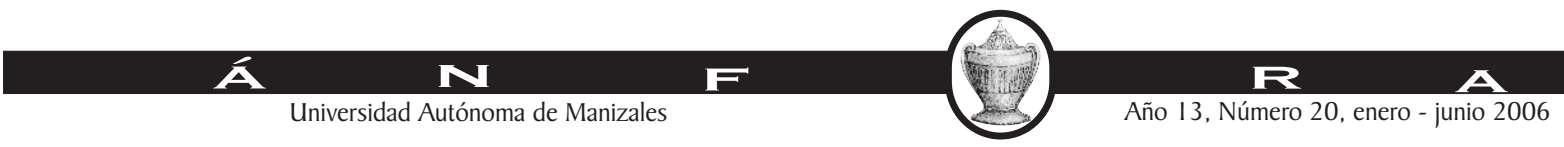


- Se ha dedicado más tiempo a la capacitación para la enseñanza de inglés general que para el trabajo con ESP.

De acuerdo con los resultados obtenidos a través de la sistematización de los talleres de capacitación docente desarrollados en la UAM, se recomendó primordialmente desarrollar más talleres enfocados al trabajo con el modelo ESP (lo cual ya se empezó a implementar), pues esto se constituye como necesidad latente ya que este modelo es la base de la metodología para la enseñanza - aprendizaje del inglés en esta universidad. Por otro lado existe el hecho de que cada año ingresan docentes nuevos al Departamento y éstos en su mayoría no tienen una formación previa respecto al trabajo con el modelo ESP.

De otro lado también se hace necesario continuar la recopilación tanto de las memorias de los talleres, como de los materiales suplementarios utilizados durante las sesiones lo que facilita la continuación, enriquecimiento y perduración del trabajo que se ha iniciado con este proyecto.

Como se planeó inicialmente, el estudio que se acaba de finalizar es la primera fase de una investigación constituida por dos fases, por lo tanto en este momento ya se ha iniciado el trabajo con la segunda fase, la cual constituye la validación de la Metodología ESP en la UAM.

\section{BIBLIOGRAFIA}

- A Forum anthology Selected articles from the English Teaching Forum 19841988.

- Aboderin Yemi, Teaching Writing. New \& ideas, English Teaching Forum, p 37 38, July 1984.

- Anderson Anne Listening. Oxford University Press. 1991.

- Bastidas Jesús A. Opciones Metodológicas para la enseñanza de Idiomas. JABA, ediciones. Pasto, Colombia 1993.

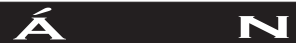

Universidad Autónoma de Manizales 
- Becerril, S COMPRENDER LA PRACTICA DOCENTE. Instituto Tecnológico de Queretano. México, 1999.

- Boughey Chrissie. Learning to write by Writing to learn, a group world approach, El T journal volume 51/2 April 1997. Oxford University Press 1997.

- Cantony Harvey, Gina. Content Area Language Instruction. Approaches and Strategies. Addison Wesley Publishing Company, 1987

- Casarini Ratto TEORIA Y DISEÑO CURRICULAR. Editorial Trillas. México 1997.

- Celce- Murcia Marianne, Teaching English as a Second or Foreign Language, Newburg house Publishers Inc. U:S:A 1979.

- Celce - Murcia Marianne. Teaching English As second or foreign Language Lois McIntosh, Editors. Newbury House Publisher, Inc USA 1989.

- Codidge Olivia, Winston Churchill and the story of two world wars

- Diaz Barriga y Hernandez, G ESTRATEgIAS DOCENTES PARA UN APRENDIZAJE SIGNIFICATIVO. Mac graw Hill. México 1998.

- FORUM, July-September 1998.

- H. Douglas Brown PRINCIPLES OF LANGUAGE LEARNING AND TEACHING. 1994.

- H. Douglas Brown TEACHING BY PRINCIPLES. An interactive approach to language pedagogy. Prentice hall. New Jersey 1994.

- Hutchinson Tom and Alan Waters, English for specific Purposes, A learning centered approach. Cambridge University Press, 1986. 
- KITAO KATHLEEN and KITAO KENI1. The history of English Teaching Methodology. www.ling.lancs.ac.uk/staff/visitors/kenji/kitao/teslhis.htm

- Ley 115 de 1994. EL LIBRO DEL TRABAJO DEL LECTOR. Art. 73

- Mohan A, Bernard Language and Content. Addison Wesley. Massachusets, 1986

- Monereo, ESTRATEgiAs DE ENSEÑANZA Y APRENDIZAJE. Graó. Barcelona, 1998.

- Nuttall Christine TEACHING READING SKILLS IN A FOREIGN LANGUAGE. Heinemann. England 1982

- Nuttal Christine. TEACHING READING SKILLS IN A FOREIGN LANGUAGE, Heinemann, 1982

- O. MALLEY J. Michael/Valdez Pierce Lorraine. AUTHENTIC ASSESSMENT FOR ENGLISH LEARNERS". Addison-Wesley. 1996.

- PERGamon. Principles and Practice in Second Language Acquisition. 1982

- Rost Longmal; Michanel. Listening in language Learning Longman Group uk, 1996.

- Rosr, Michael. Introducing listening. Penguin English Applied Linguistics, 1994.

- Sion Christopher, More Recipes for Tired Teachers. Well Seasoned Activities for the ESOL Classroom. Addison Wesley Publishing Company, inc. pg 111, 1991.

- Snow Marguerite Ann et al. A conceptual Framework for the integration of language and content in second Foreign Language Instruction. TESOL 
QUATERLY Vol. 0 número 2 June.

- Underwood Mary. Teaching Listening Longman Handbooks Language Teachers. London 1990 